\title{
Performance Comparison of Adaptive filtering Algorithms for Parallel Interference Cancellation in Asynchronous MC DS-CDMA Systems
}

\author{
Kh. El-Barbary ${ }^{*}$, H.E. Abou- Bakr ${ }^{\dagger}$, and M. Ahmed ${ }^{\ddagger}$
}

\begin{abstract}
The Bit Error Rate (BER) comparison of Adaptive Parallel Interference Cancellation (APIC) algorithms in asynchronous Multicarrier Direct Sequence Code Division Multiple Access (MC-DS-CDMA) system is introduced. The APIC is used to eliminate the Multiple Access Interference (MAI) in MC-DS-CDMA system. The Adaptive filtering algorithms considered are, Normalized Least Mean Squares (NLMS), Variable Step Size Normalized Least Mean Squares (VSS-NLMS), and Generalized Normalized Gradient Descent (GNGD) algorithms. These algorithms are used in coincidence with APIC system for updating cancellation weights. The performance comparison between these algorithms is performed via extensive computer simulations. Simulation results demonstrate that the VSSNLMS algorithm outperforms all other algorithms, the NLMS is modular, while the worst performance is obtained by GNGD.
\end{abstract}

Keywords: MC DS-CDMA, Adaptive filtering, Parallel interference cancellation,

\section{Introduction}

Multicarrier approach offers several advantages, including robustness in fading and interference environments, operation at lower chip rates and non-contiguous bandwidth operation [1]. The multicarrier CDMA schemes are categorized mainly into two groups, first, MC-CDMA scheme that spreads the original data stream over different subcarriers using a given spreading code in the frequency domain [1], while the other group, MC DS-CDMA spreads the serial-to-parallel (S/P) converted data streams using a given spreading code in the time domain [2].

It is well known that the major concern in multiple access systems is Multiple Access Interference (MAI), because it limits the maximum available capacity in terms of the number of users. Multiuser Detectors (MUD) for code division multiple access (CDMA) receivers are effective techniques to eliminate the MAI. It is well known that the prohibitive complexity of the optimal multiuser detection necessities suboptimal solutions having lower complexity. Many suboptimal MUD algorithms have been proposed in the literature, which can be classified into two major approaches, namely, adaptive filtering [3] and interference cancellation (IC) [4]. Much more researches have been dedicated to the latter primarily due to

\footnotetext{
* Modern Academy, Staff member, khbar2000@ yahoo.com.

†Egyptian Armed Forces, hossameldin_aboubakr@ hotmail.com

*Egyptian Armed Forces, maged-mohammed@excite.com. 
simpler analysis tractability. There are two main varieties of IC schemes, serial IC (SIC), and parallel IC (PIC), the latter is discussed here. PIC is classified into two groups namely, Soft Decision PIC (SD PIC), and Hard Decision PIC (HD PIC), under assumption of perfect channel estimation the HD PIC outperforms the SD PIC.

For further performance improvement of PIC system, adaptive PIC (APIC) scheme has been proposed for DS-CDMA system [5], and for MC-DS-CDMA system [6]. In each stage of the APIC receiver, MAI estimates are obtained using the bit estimates from the previous stage and the adaptive weights for the bit estimates. The adaptive weights are obtained by minimizing the Euclidean distance between the received signal and its estimate through different types of adaptive filter algorithms.

In this paper, different approaches for updating cancellation weights are analyzed. These algorithms are NLMS, VSS-NLMS, and GNGD. The performance of APIC using NLMS is examined analytically and the results are verified using computer simulation. In addition other algorithms VSS-NLMS, and GNGD are examined for asynchronous MC DS-CDMA system over a frequency selective Rayleigh fading channels using computer simulations.

The rest of this paper is organized as follows: in Section 2 the MC DS-CDMA system model is introduced, its performance using Conventional PIC (CPIC), and APIC are analyzed in Sections 3. In Section 4, three generalized square-error regularized LMS algorithms are summarized. In Section 5, performance comparison of the APIC with different adaptive filtering schemes is performed through computer simulations. Finally, Section 6 summarizes the main conclusions of the paper.

\section{System Model}

Asynchronous MC DS-CDMA system with $K$ users communicate simultaneously at the same rate is considered. The transmitter spreads the $\mathrm{S} / \mathrm{P}$ converted data streams using a given spreading code in the time domain, so that the resulting spectrum of each subcarrier can satisfy the orthogonality condition with a minimum frequency separation [7]. This scheme was originally proposed for an uplink communication system, because the characteristics of the orthogonal MC DS-CDMA are effective for establishing a quasi-synchronous channel.[7]. A brief description of the transmitter, channel model, and receiver is presented in the next subsections.

\subsection{Transmitter}

The transmitter block diagram for the MC DS-CDMA system is shown in Fig. (1). In this scheme the initial data stream having the bit duration of $T_{b}$ is $\mathrm{S} / \mathrm{P}$ converted to $p$ number of lower-rate substreams. Hence, the new bit duration after the S/P conversion or the symbol duration is $T_{s}=p T_{b}$. Each of the $p$ lower-rate substreams is spread by the time-domain spreading PN code $c^{k}(t)$, with length $N$.Each of the $p$ substreams is transmitted by $M$ number of subcarriers, in order to achieve a frequency diversity order of $M$. Maximal Ratio Combining (MRC) technique is used at the receiver to exploit the frequency diversity inherent in the Multicarrier (MC) system for combating effects of channel fading.

Hence, the total number of subcarriers required by the MC DS-CDMA system is $U=p M$. Based on this, the transmitted signal of $k^{\text {th }}$ can be modeled as

$$
\mathrm{s}_{\mathrm{k}}(t)=\sum_{i=1}^{P} \sum_{j=1}^{M} \sqrt{\frac{2 P_{k}}{M}} b_{i}^{(k)}(t) c^{(k)}(t) \cos \left(2 \pi f_{i j} t+\phi_{i j}^{k}\right)
$$


where $P_{k}$ is the transmitted power of the $k^{\text {th }}$ user, $b_{i}^{(k)}(t)=\sum_{-\infty}^{\infty} b_{i}^{k}[n] P_{T_{s}}\left(t-n T_{s}\right), i=1,2, . ., p$ represents the binary data of the $i^{\text {th }}$ substream, where $b_{i}^{k}[n]$ is assumed to be random variable taking value of +1 or -1 with equal probability, while $P_{T_{s}}(\mathrm{t})$ represents the rectangular shape waveform, and $c^{k}(t)$ represents the T-domain spreading code assigned to the $k^{\text {th }}$ user, which is the same for all the $U=p M$ number of subcarriers. The spreading sequence $c^{k}(t)$ can be expressed as $c^{k}(t)=\sum_{-\infty}^{\infty} c_{j}^{k} P_{T_{c}}\left(t-j T_{c}\right)$, where $c_{j}^{k}$ assumes values of +1 or -1 , while $P_{T_{c}}$ is the chip waveform of the $\mathrm{T}$ domain spreading sequence, which is defined over the interval $\left[0, T_{c}\right)$. Finally, $\phi_{i j}^{k}$ represents the initial phase associated with the carrier modulation in the context of the subcarrier $(i, j)$ in (1).In MC DS-CDMA systems subcarrier signals are chosen to be orthogonal to each other with the minimum frequency separation after the DS spreading, it can be shown that the minimum spacing between two adjacent subcarriers satisfies $\Delta f=1 / T_{c}$, which results in a successively half-overlapping. It can be shown that, for binary spreading sequences having a chip duration of $T_{c}, W_{D S}$ (represents the null-to-null bandwidth of each spread subcarrier signal) can be expressed as $W_{D S}=\frac{2}{T_{c}}$.

\subsection{Channel Model}

The channel is assumed to be a slow varying frequency-selective Rayleigh fading channel with a delay spread of $T_{m}$. It has been shown that the principle motivation for using MC-CDMA is to allow a frequency selective fading channel to appear as flat fading on each subcarrier [7]. If the number of sub-carriers satisfies $p M \geq 2 L_{1}-2$ where $L_{1}$ is the number of resolvable paths for single carrier case, then each sub-carrier undergoes a single path fading channel. With this assumption, the complex channel gain for the subcarrier $(i, j)$ and $k^{\text {th }}$ user can be defined as [6].

$$
\zeta_{i j}^{k}(t)=\alpha_{i j}^{k}(t) \exp \left(j \psi_{i j}^{k}(t)\right)
$$

\subsection{Receiver}

Assuming that there are $K$ asynchronous users in the MC DS-CDMA system, where all of them use the same $U=p M$ subcarriers, the average power received from each user at the base station is also assumed to be the same, implying perfect power control. When the transmitted signal is in the form of (1), the received signal at the base station can be expressed as [6],

$$
r(t)=\sum_{k=1}^{K} \sum_{i=1}^{p} \sum_{j=1}^{M} \sqrt{\frac{2 P}{M}} \alpha_{i j}^{k} b_{i}^{k}\left(t-\tau_{k}\right) c_{k}\left(t-\tau_{k}\right) \cos \left(2 \pi f_{i j} t+\varphi_{i j}^{k}\right)+n(t)
$$

where $\tau_{k}$ represents the time mismatch in the context of the $k^{\text {th }}$ user, with respect to the reference user, $\alpha_{i j}^{k}$ is the channel fading coefficient, $\varphi_{i j}^{k}=\phi_{i j}^{k}-\psi_{i j}^{k}-2 \pi f_{i j} \tau_{k}$, which is assumed to be an independent identically distribution (i.i.d) random variable having a uniform distribution in $[0,2 \pi), \psi_{i j}^{k}$ is due to the transmission channel, while $n(t)$ represents the AWGN with zero mean and double-sided PSD of variance $N_{0} / 2$. Assuming that the first user $k=1$ is the reference user. The correlation receiver block diagram of the reference user is shown in Fig. (2), where the superscripts and subscripts concerning the reference user have been omitted for the sake of simplicity. The receiver provides a correlator for each subcarrier 
and the correlator outputs associated with the same data bit are combined to form a decision variable. Finally, a P/S converter is employed to recover the serial data stream. For MRC, the decision variable for detecting $b_{u}$ can be written as $Z_{u}=\sum_{v=1}^{M} Z_{u v}$

where

$$
\begin{aligned}
Z_{u v} & =\int_{0}^{T_{s}} r(t) g_{u v} c(t) \cos \left(2 \pi f_{u v} t\right) d t \\
Z_{u v} & =D_{u v}+N_{u v}+\sum_{k=2}^{K} I_{1}^{(k)}+\sum_{\substack{k=2 \\
k}}^{K} \sum_{\substack{j \neq 1 \\
j \neq v i v=u}}^{p} \sum_{j=1}^{M} I_{2}^{(k)}(i, j) \\
D_{u v} & =\int_{0}^{T_{s}} \sqrt{\frac{2 P}{M}} \alpha_{u v} b_{u}(t) c(t) \cos \left(2 \pi f f_{u v} t+\varphi_{u v}\right) g_{u v} c(t) \cos \left(2 \pi f_{u v} t\right) d t
\end{aligned}
$$

where $g_{u v}=\alpha_{u v}$ is assumed, associated with perfect channel estimation, MRC diversity combining scheme, $\varphi_{u v}=0$, and $\tau_{1}=0$ then the desired signal (5) can be written as,

$$
D_{u v}=\sqrt{\frac{2 P}{M}} \frac{\alpha_{u v}^{2}}{2} \int_{0}^{T_{s}} b_{u}(t) d t=\sqrt{\frac{2 P}{M}} \frac{g_{u v}^{2}}{2} b_{u} T_{s},
$$

while the noise term can be expressed as

$$
N_{u v}=\int_{0}^{T_{s}} n(t) g_{u v} c(t) \cos \left(2 \pi f_{u v} t\right) d t .
$$

Multiuser interference term $I_{1}^{(k)}$ represents the interference from the other users at the same considered subcarrier, i.e. the subcarrier determined by $(u, v)$, can be written as,

$$
\begin{aligned}
I_{1}^{(k)} & =\sqrt{\frac{2 P}{M}} \frac{\alpha_{u v}^{k} g_{u v}}{2} \cos \left(\varphi^{k}{ }_{u v}\right) \int_{0}^{T_{s}} b_{u}^{k}\left(t-\tau_{k}\right) c_{k}\left(t-\tau_{k}\right) c(t) d t \\
& =\sqrt{\frac{P}{2 M}} \alpha_{u v}^{k} g_{u v} \cos \left(\varphi^{k}{ }_{u v}\right)\left[b_{u}^{k}[-1] R_{k 1}\left(\tau_{k}\right)+b_{u}^{k}[0] \hat{R}_{k 1}\left(\tau_{k}\right)\right]
\end{aligned}
$$

where, $R_{k 1}\left(\tau_{k}\right), \hat{R}_{k 1}\left(\tau_{k}\right)^{\S}$ are the partial cross-correlation function between the $k^{\text {th }}$ user's spreading sequence waveform $c_{k}\left(t-\tau_{k}\right)$ and the reference user's spreading sequence waveform $c(t)$ [7].

Finally, the last term in (4), the multiuser interference term $I_{2}{ }^{k}$ is due to the other users from other subcarriers $(i \neq u, j \neq v)$ which can be expressed as.

$$
I_{2}^{k}(i, j)=\sqrt{\frac{P}{2 M}} \alpha_{u v}^{k} g_{u v} \int_{0}^{T_{s}} b_{u}^{k}\left(t-\tau_{k}\right) c_{k}\left(t-\tau_{k}\right) c(t) \cos \left(2 \pi\left(f_{i j}-f_{u v}\right) t+\phi_{i j}^{k}\right) d t
$$

\section{Performance Analysis of HD-CPIC, and HD-APIC}

\subsection{HD-CPIC Stage}

For the HD-CPIC receiver shown in Fig. (2), the interference cancellation is performed by subtracting the estimated signals of the interfering users from the reference signal $r_{u v}(t)$ that can be written as

\footnotetext{
${ }^{\S}$ the "hat" notation on the cross-correlation functions is used to denote the correlation over the complementary (with respect to the symbol duration) portion of the integration interval.
} 


$$
\begin{aligned}
r_{u v}(t)= & \sqrt{\frac{2 P}{M}}\left[\sum_{k=1}^{K} \frac{\alpha_{u v}^{k}}{2} \cos \left(\phi_{u v}^{k}\right) b_{u}^{k}\left(t-\tau_{k}\right) c_{k}\left(t-\tau_{k}\right)+\right. \\
& \left.\sum_{k=1}^{K} \sum_{\substack{i=1 \\
i f i=u}}^{p} \sum_{\substack{j=1 \\
j \neq v}}^{M} \alpha_{i j}^{k} b_{i}^{k}\left(t-\tau_{k}\right) c_{k}\left(t-\tau_{k}\right) \cos \left(2 \pi f_{i j} t+\varphi_{i j}^{k}\right) \cos \left(2 \pi f_{u v} t\right) d t\right]+\eta_{n}(t)
\end{aligned}
$$

This subtraction results in a cleaner signal which is used to get more accurate decision for the desired user assuming perfect channel estimation, the reconstructed signal for subcarrier $u v$ and user $k$ can be expressed as,

$$
\hat{I}_{1}^{(k)}=\sqrt{\frac{2 P}{M}} \frac{g_{u v}^{k}}{2} \cos \left(\phi_{u v}^{k}\right) \hat{b}_{u}^{k}\left(t-\tau_{k}\right) c_{k}\left(t-\tau_{k}\right),
$$

while, the reconstructed signal from other subcarriers $\hat{I}_{2}^{(k)}$ is defined as,

$$
\left.\hat{I}_{2}^{(k)}=\sqrt{\frac{2 P}{M}} \frac{g_{i j}^{k}}{2} \hat{b}_{i}^{k}\left(t-\tau_{k}\right) c_{k}\left(t-\tau_{k}\right) \cos \left[2 \pi\left(f_{i j}-f_{u v}\right) t+\phi_{i j}^{k}\right)\right]
$$

where $\hat{b}_{u}^{k}$ is the estimate of the transmitted $u^{\text {th }}$ bit for the $k^{\text {th }}$ user, while $\phi_{u v}^{k}$ is a random variable with a uniform distribution $[0,2 \pi)$. Giving that the first user $k=1$ is the reference user, then the decision variable for detecting $b_{u v}^{(1)}$ for the $u v^{\text {th }}$ subcarrier (the superscript here represents first PIC stage) can be expressed as,

$$
\begin{aligned}
\hat{z}_{u v}^{1} & \left.=\int_{0}^{T_{s}}\left\{r_{u v}(t)-\left[\sum_{k=2}^{K} \hat{I}_{1}^{(k)}+\sum_{k=2}^{K} \sum_{\substack{i=1 \\
i f=u}}^{P} \sum_{\substack{j=1 \\
j \neq v}}^{M} \hat{I}_{2}^{(k)}\right]\right\} c(t) g_{u v}^{1}\right\} d t \\
& =D_{u v}+N_{u v}+\sum_{k=2}^{K} v_{1}^{(k)}+\sum_{k=2}^{K} \sum_{\substack{i=1 \\
i f i=u}}^{P} \sum_{\substack{j=1 \\
j \neq v}}^{M} v_{2}^{(k)}
\end{aligned}
$$

The first and second terms $D_{u v}$, and $N_{u v}$ represent the desired and noise terms respectively same as defined before in (6), (7), however the last two terms in (13) represent the residual multiuser, and residual multicarrier interference respectively. $v_{1}^{(k)}$ can be written as

$$
v_{1}^{(k)}=\int_{\tau_{k}}^{\tau_{k}+T s} \sqrt{\frac{2 P}{M}} \frac{\alpha_{u v}^{k} g_{u v}^{1}}{2} \cos \left(\phi_{u v}^{k}\right)\left[b_{u}^{k}\left(t-\tau_{k}\right)-\hat{b}_{u}^{k}\left(t-\tau_{k}\right)\right] c_{k}\left(t-\tau_{k}\right) c(t) d t
$$

Hence, the variance of $v_{1}^{(k)}$ can be written as [8],

$$
\operatorname{var}\left(v_{1}^{(k)}\right)=E\left[\left(\sqrt{\frac{2 P}{M}} \frac{\alpha_{u v}^{k} g_{u v}^{1}}{2} \cos \left(\phi_{u v}^{k}\right)\right)^{2}\right] E\left(\left(\chi_{u}^{(k)}\right)^{2}\right) E\left(\left[R_{K 1}\left(\tau_{k}\right)+\hat{R}_{K 1}\left(\tau_{k}\right)\right]^{2}\right.
$$

where $\chi_{u}^{(k)}=\left(b_{u}^{k}[-1]-\hat{b}_{u}^{k}[-1]\right), \chi_{u}^{\prime(k)}=\left(b_{u}^{k}[0]-\hat{b}_{u}^{k}[0]\right)$, and $\quad E\left(\left(\chi_{u}^{(k)}\right)^{2}\right)=E\left(\left(\chi_{u}^{(k)}\right)^{2}\right)=4 p_{\text {ini }}(e)$, given that $P_{i n i}(e)$ is the BER of the first stage (MF-MRC). It has been shown for rectangular chip waveforms, and random spreading code the variance of continuous-time partial crosscorrelation can be approximated as [8].

$$
E\left(\left[R_{K 1}\left(\tau_{k}\right)+\hat{R}_{K 1}\left(\tau_{k}\right)\right]^{2}\right)=\frac{2 T_{s}^{2}}{3 N}
$$

Then, from (15), and (16), the variance of multiuser interface $v_{1}^{(k)}$ can be written as

$$
\operatorname{var}\left(v_{1}^{(k)}\right)=\frac{P}{2 M} g^{2}{ }_{u v} \frac{\Omega}{2} \frac{2 T_{s}^{2}\left(4 P_{i n i}(e)\right)}{3 N}=\frac{P}{M} \frac{\Omega g^{2}{ }_{u v}}{6 N}\left(4 P_{i n i}(e)\right) T_{s}^{2}
$$

where $\Omega=E\left(\left(\alpha_{u v}^{k}\right)^{2}\right)$

Similarly for the second interference term $v_{2}^{(k)}$, which can be written as

$$
v_{2}^{(k)}=\sqrt{\frac{2 P}{M}} \sum_{k=2}^{K} \sum_{\substack{i=1 \\ i f}}^{p} \sum_{j=u}^{M} \alpha_{j \neq v}^{k} g_{u v}^{1} \int_{0}^{T_{s}}\left[b_{u}^{k}\left(t-\tau_{k}\right)-\hat{b}_{u}^{k}\left(t-\tau_{k}\right)\right] c_{k}\left(t-\tau_{k}\right) c(t) \cos \left(2 \pi\left(f_{i j}-f_{u v}\right) t+\phi_{i j}^{k}\right)
$$


which is a zero mean RV and its variance is obtained by modification of [7] ${ }^{* *}$, which can be written as,

$$
\operatorname{var}\left(v_{2}^{(k)}\right)=\frac{P}{2 M} \Omega g^{2}{ }_{\mu v} \frac{T_{s}^{2}\left(4 P_{i n i}(e)\right)}{2 \pi^{2}[(i-u)+(j-v) p]^{2}}[1-\operatorname{sinc}(2 \pi([(i-u)+(j-v) p]))]
$$

The mean of the decision variable for the CPIC, $\hat{Z}_{u}^{(1)}$ is given by $E\left[\hat{Z}_{u}^{(1)}\right]=\sqrt{\frac{P}{2 M}} b_{u} T \sum_{v=1}^{M} g_{u v}^{2}$ while its variance is the sum of variances of all interference terms and noise, according to (17), (19) it can be expressed as,

$$
\operatorname{Var}\left[\hat{Z}_{u}^{(1)}\right]=\left[\frac{N_{0}}{4} T_{s}+\frac{P \Omega}{M} \frac{(k-1)}{6 N}\left(4 P_{i n i}(e)\right) T_{s}^{2}+\frac{P \Omega}{2 M}(k-1) M(p-1)\left(4 P_{i n i}(e)\right) T_{s}^{2} \bar{I}_{o}\right] \sum_{v=1}^{M} g_{u v}^{2}
$$

where $\bar{I}_{o}$, represents the average of $\operatorname{var}\left(v_{2}^{(k)}\right)$ which can be written as [7]

$$
\begin{aligned}
& \bar{I}_{o}=\frac{1}{p M} \sum_{u=1}^{p} \sum_{v=1}^{M} I(u, v) \\
& I(u, v)=E_{i, j}\left[\operatorname{Var}\left(I_{2}^{k}\right)\right] \\
& \quad=\frac{p \Omega}{2 M} \frac{1}{p(M-1)} \sum_{i=1}^{p} \sum_{\substack{j=1 \\
j \neq v}}^{M} \frac{T_{s}^{2} i=u}{2 \pi^{2}[(i-u)+(j-v) p]^{2}}[1-\operatorname{sinc}(2 \pi([(i-u)+(j-v) p])
\end{aligned}
$$

Assuming that any bit can be sent via any of the $p$ parallel branches with equal probability, the final BER of the CPIC-MRC receiver can be written as [9],

$$
p_{p i c}^{(1)}[e]=\left[\frac{1}{2}\left(1-\frac{1}{\sqrt{W^{2}+1}}\right)\right]^{M} \sum_{u=0}^{M-1}\left(\begin{array}{c}
M-1+u \\
u
\end{array}\right)\left[\frac{1}{2}\left(1+\frac{1}{\sqrt{W^{2}+1}}\right)\right]^{u}
$$

where $\gamma=\frac{\chi}{2}, W=\left(\frac{\sqrt{2 \operatorname{Var}\left[\hat{Z}_{(1)}\right]}}{E\left[\hat{Z}_{(1)}\right]}\right)$

\subsection{HD-APIC Stage}

For HD-APIC, the interference cancellation is quite similar HD-CPIC except that the estimated signals of the interfering users are weighted before subtracted from reference signal $r_{u v}(n)$. For subcarrier $u v$, the output from APIC scheme can be written as

$$
x_{u, v}^{(1)}(n)=r_{u v}(n)-\sum_{k=2}^{K} \hat{I}_{1}^{(k)} w_{u v}^{(k)}-\sum_{k=2}^{K} \sum_{\substack{i=1 \\ i f}}^{P} \sum_{\substack{j=1 \\ j=1}}^{M} \hat{I}_{2 \neq v}^{(k)} w_{i j}^{(k)}
$$

where $w_{u v}^{(k)}$, and $w_{i j}^{(k)}$ are the weights for subcarrier $u v$, and subcarrier $i j$ respectively for the $k^{\text {th }}$ user. Adaptive weights are updated at the chip rate according to the selected adaptive algorithm as shown in Fig. (3). The weights updating equations for different adaptive algorithms will be discussed in the next section.

The performance of HD-APIC can be derived as follows, let

$$
\Delta r_{u v}(n)=r_{u v}(n)-\sum_{k=1}^{K} \hat{I}_{1}^{(k)} w_{u v}^{(k)}-\sum_{k=1}^{K} \sum_{\substack{i=1 \\ i f i=u}}^{P} \sum_{\substack{j=1 \\ j \neq v}}^{M} \hat{I}_{2}^{(k)} w_{i}^{(k)}
$$

be the difference between the reference signal $r_{u v}(n)$ and the composite estimated weighted signals from other users, and subcarriers. In Fig. (2) the output from the PIC for the desired user, and $u v$ subcarrier is given by[6].

$$
x_{u, v}^{(1)}(n)=r_{u v}(n)-\sum_{k=1}^{K} I_{1}^{(k)}(n)-\sum_{k=1}^{K} \sum_{\substack{i=1 \\ i f \mathfrak{i} i=u}}^{P} \sum_{\substack{j=1 \\ j \neq v}}^{M} \widetilde{I}_{2}^{(k)}(n)+\tilde{I}_{1}^{(1)}(n)
$$


which can be written as $x_{u, v}^{(1)}(n)=\Delta r_{u v}(n)+\tilde{I}_{1}^{1}(n)$,

$$
=\Delta r_{u v}(n)-\Delta I_{1}^{(1)}(n)+I_{1}^{(1)}(n),
$$

where

$$
\Delta I_{1}^{(1)}(n)=I_{1}^{(1)}(n)-\tilde{I}_{1}^{(1)}(n) .
$$

The soft output of the APIC-MRC stage is given by

$$
\begin{aligned}
\tilde{z}_{u}^{1}(n) & =\Re\left\{\sum_{v=1}^{M} x_{u v}^{(1)}(n) c(n)\left[g_{u v}^{1}\right]^{*}\right\} \\
& =\sum_{v=1}^{M} I_{1}^{(1)}(n) c(n)\left[g_{u v}^{1}\right]^{*}+\sum_{v=1}^{M}\left[\Delta r_{u v}(n)-\Delta I_{1}^{(1)}(n)\right] c(n)\left[g_{u v}^{1}\right]^{*}
\end{aligned}
$$

The first term in the RHS of Equation (26) represents the desired signal $D$, the second term is the interference $I$, which is approximated as a zero mean Gaussian random variable, with variance given by[6]

$$
\operatorname{var}(I)=\sum_{v=1}^{M} g_{u v}^{2} \cdot \frac{(K-1) E\left[\left(\Delta r_{u v}(n)\right)^{2}\right]+\sigma_{\eta}^{2}}{2 K}
$$

where $E\left[\left(\Delta r_{u v}(n)\right)^{2}\right]$ is the mean square error (MSE) of the MAI estimation which can be calculated with the aid of computer simulation [6], then the BER can be calculated as stated before in CPIC stages.

\section{Generalized Square-Error Regularized LMS Algorithm}

Several adaptive algorithms, including NLMS, GNGD, and VSS-NLMS are summarized. These algorithms can be used with the APIC system to update weights to eliminate the MAI of the MC DS-CDMA.

\subsection{Conventional Regularization for NLMS (\&-NLMS) [10 ]}

Let $\mathbf{w}(n)$ denote the coefficient vector of the update weight at iteration $n$. $\mu$ is the step size The a priori estimation error between the desired response and the output of the LMS filter is $e(n)=d(n)-X^{T}(n) \mathbf{W}(n)$.

The $\varepsilon$-NLMS algorithm updates $\mathbf{w}(\mathbf{n})$ as follows

$$
\mathbf{w}(n+1)=\mathbf{w}(n)+\frac{\mu}{\left(\|\mathbf{x}(n)\|^{2}+\varepsilon\right.} e(n) \mathbf{x}(n)
$$

where $\varepsilon$ is a fixed small positive constant called regularization parameter. The overall effective step-size might become relatively large or relatively small, Depending on the value of $\varepsilon$ and this affects the convergence and tracking performance.

\subsection{Generalized Normalized Gradient Descent (GNGD) Algorithm [10]}

The GNGD algorithm belongs to the family of time-varying regularized VSS algorithm. The filter coefficient vector is updated as

$$
\mathbf{w}(n+1)=\mathbf{w}(n)+\frac{\mu_{c}}{\left(\|\mathbf{x}(n)\|^{2}+\varepsilon(n)\right.} e(n) \mathbf{x}(n)
$$

where $\mu_{c}$ is a fixed step size. The regularization parameter $\varepsilon(n)$ is recursively calculated as

$$
\varepsilon(n)=\varepsilon(n-1)-\rho \mu_{c} \cdot \frac{e(n) e(n-1) \mathbf{x}^{T}(n) \mathbf{x}(n-1)}{\left(\|\mathbf{x}(n-1)\|^{2}+\varepsilon(n-1)\right)^{2}}
$$

where $\rho$ is an adaptation parameter, and $\varepsilon(0)$ is the initial value of the regularization parameter. 


\subsection{Variable Step-Size NLMS (VSS-NLMS) Algorithm [10]}

A variable step size NLMS (VSS-NLMS) was obtained as follows.

$$
\begin{aligned}
& \mathbf{P}(n)=\beta(n-1)+(1-\beta) \mathbf{x} \cdot(n)\left(\mathbf{x}^{T}(n) \mathbf{x} \cdot(n)+\delta\right)^{-1} e(n) \\
& \mu(n)=\mu_{\max } \cdot \frac{\|p(n)\|^{2}}{\|p(n)\|^{2}+\delta_{2}}
\end{aligned}
$$

The filter coefficient vector update recursion is given by

$$
\mathbf{w}(n+1)=\mathbf{w}(n)+\mu(n) \mathbf{x} \cdot(n)\left(\mathbf{x}^{T}(n) \mathbf{x} \cdot(n)+\delta_{1}\right)^{-1} e(n)
$$

The squared instantaneous a priori estimation error is used to update the step size as $\mu(n+1)=\alpha \mu(n)+\gamma$

where $0<\alpha<1, \gamma>0$, and $\mu(n+1)$ is restricted in some pre-decided $\left[\mu_{\min }, \mu_{\max }\right]$.

\section{Simulation Results}

In this section, the bit error rate performance of the asynchronous MC-DS-CDMA system HD-APIC using three different adaptive algorithms is studied. The considered channel is a Rayleigh frequency-selective fading channel. For each sub-carrier the channel is considered flat. To achieve a frequency diversity, $M$ is set to be equal to 2 . PN random sequences are generated as spreading codes with different lengths that equal to $N=31$, and $N=63$. The total transmitted power is the same irrespective of the number of subcarriers, and all users are received with the same power (perfect power control system is assumed). Extensive computer simulations have been done to compare between the presented adaptive algorithms. The simulation results were obtained with the following setup:

$$
\beta=0.99, \delta=0.1, \delta 2=0.001, \mu=0.2, \mu_{\text {min }}=0.001, \mu_{\max }=1, \mu_{c}=0.1 .
$$

Bit error rate for asynchronous MC DS-CDMA with 15 active users, and 6 subcarriers is shown in Fig.(4) It is clear from the figure both the analytic and simulation results have a good agreement. The MF performance is completely decayed due to the MAI, and fading channel. The APIC is better than the CPIC specially for higher SNR $(=13 \mathrm{~dB})$.

Fig.(5) shows the excess MSE for the three adaptive algorithms at 20dB SNR. Both NLMS, and VSS-NLMS have a fast convergence than GNGD-NLMS. However, the performance of NLMS is the worst for the steady state error. Fig. (6) illustrates the performance comparison of the three adaptive algorithms in terms of BER at various values of SNR. As can be seen, VSS-NLMS gives the best performance, then NLMS while GNGD has the worst performance. This result for GNGD can be justified as the weight decision is taken at the last chip of every bit which is in this case $n=31$, as shown in Fig. (5) the GNGD algorithm has a slow convergence rate, in order to further illustrate this result, Fig.(7) shows the performance of the three algorithms using a PN code with length $N=63$. As can be seen the GNGD has a better performance than NLMS and a slight degradation than VSS-NLMS.

Figs. (8), (9) show the performance comparison in terms of BER versus the number of active users, at various values of SNR ( $E_{b} / N_{0}=15 \mathrm{~dB}$, and $25 \mathrm{~dB}$ ), $N=31$. The results agreed with the previous result as VSS-NLMS outperform both NLMS, and GNGD.

\section{Conclusions}

Three different square-error regularized LMS algorithm are tested with APIC to eliminate the MAI of an asynchronous MC DS-CDMA system over frequency selective fading channels. A closed-form expressions of the BER of asynchronous MC DS-CDMA for HD-APIC- 
NLMS receivers has been derived. A performance comparison is achieved by means of extensive computer simulations. The results show that BER performance of APIC-VSSNLMS surplus both APIC-GNGD, and APIC-NLMS.

\section{References}

[1] N.Yee, J-P. Linnartz and G. Fettweis, "Multicarrier CDMA in Indoor Wirless Radio Networks," Proc.of IEEE PIMRC' 93, Yokohama, Japan, Sept. 1993, pp.109-13.

[2] Xiang Gui, Tung Sang Ng, "Performance of Asynchronous Orthogonal Multicarrier CDMA System in Frequency Selective Fading Channel," IEEE Trans. Commun.,vol.47, no.7, Jul.1999,pp. 1084-91.

[3] M. L. Honig and H. V. Poor, "Adaptive interference suppression," in Wireless Communications: Signal Processing Perspectives, pp. 64-128, Prentice-Hall, Upper Saddle River, NJ, USA, 1998.

[4] L. Fang and L. B. Milstein, "Successive interference cancellation in multicarrier DS/CDMA," IEEE Transactions on Communications, vol. 48, no. 9, pp. 1530-1540, 2000.

[5] S.R. Kim, et al., Adaptive weighted parallel interference cancellation for CDMA systems, Electron. Lett. 34 (22) (1998) 2085-2086.

[6] H.Wang, K. Yen, K.W. Ang, and Y. H. Chew, "Design and performance analysis of an adaptive receiver for multicarrier DS-CDMA," EURASIP Journal on Wireless Communications and Networking., vol. 2007 , Issue 2 January 2007.

[7] Hanzo, L., Yang, L. L., Kuan, E. L. and Yen, K. " Single-and Multi-carrier DS-CDMA: Multi-User Detection, Space-Time Sprreading, Synchronisation, Standards and Networking," (2003), joha Wiley \& Sons.

[8] S. Hara and R. Prasad, "Overview of multicarrier CDMA," IEEE Communications Magazine, vol. 35, no. 12, pp. 126-133, 1997.

[9] J. Proakis, "Digital Communications”, McGraw-Hill, New York, NY, USA, 4th edition, 2001.

[10] J. Lee, J. W. Chen, and H.C. Huang, "Performance Comparison of Variable Step-Size NLMS Algorithms", Proceedings of the World Congress on Engineering and Computer Science 2009 Vol I, WCECS 2009, October 20-22, 2009, San Francisco, USA. 


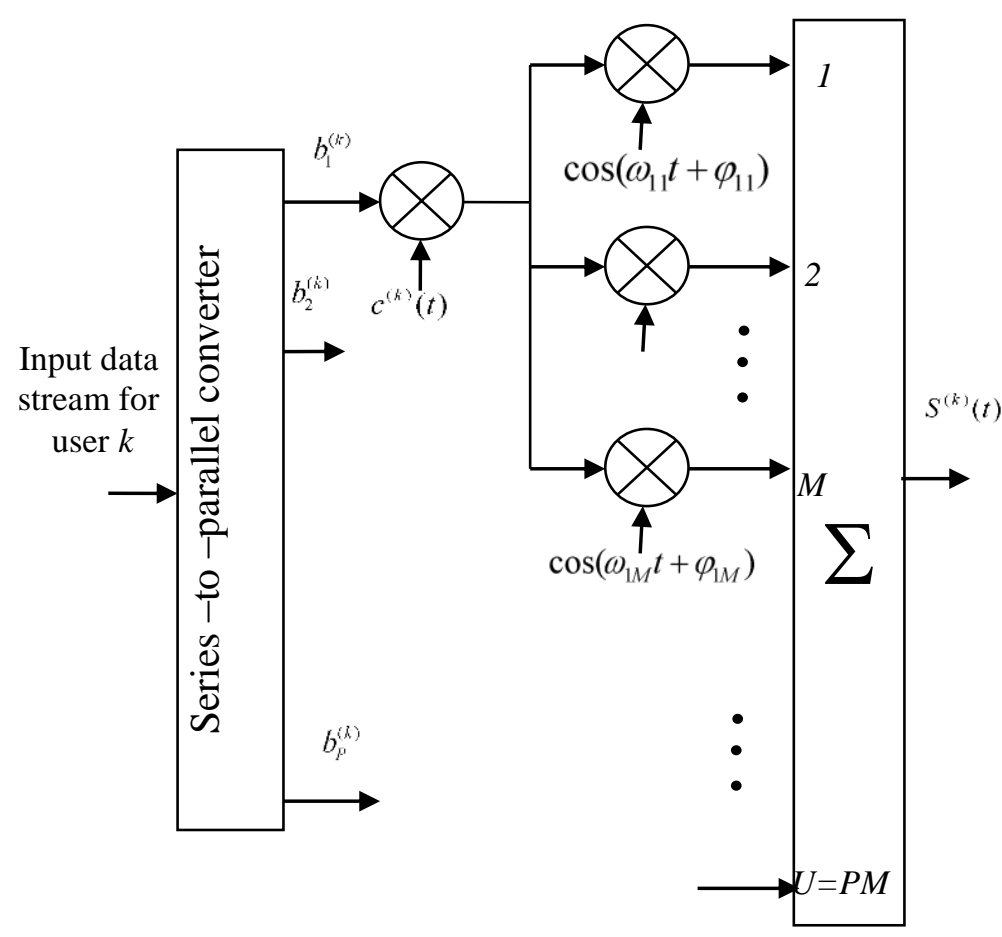

Fig. 1 Orthogonal multicarrier DS-CDMA transmitter for users $k$. [7]

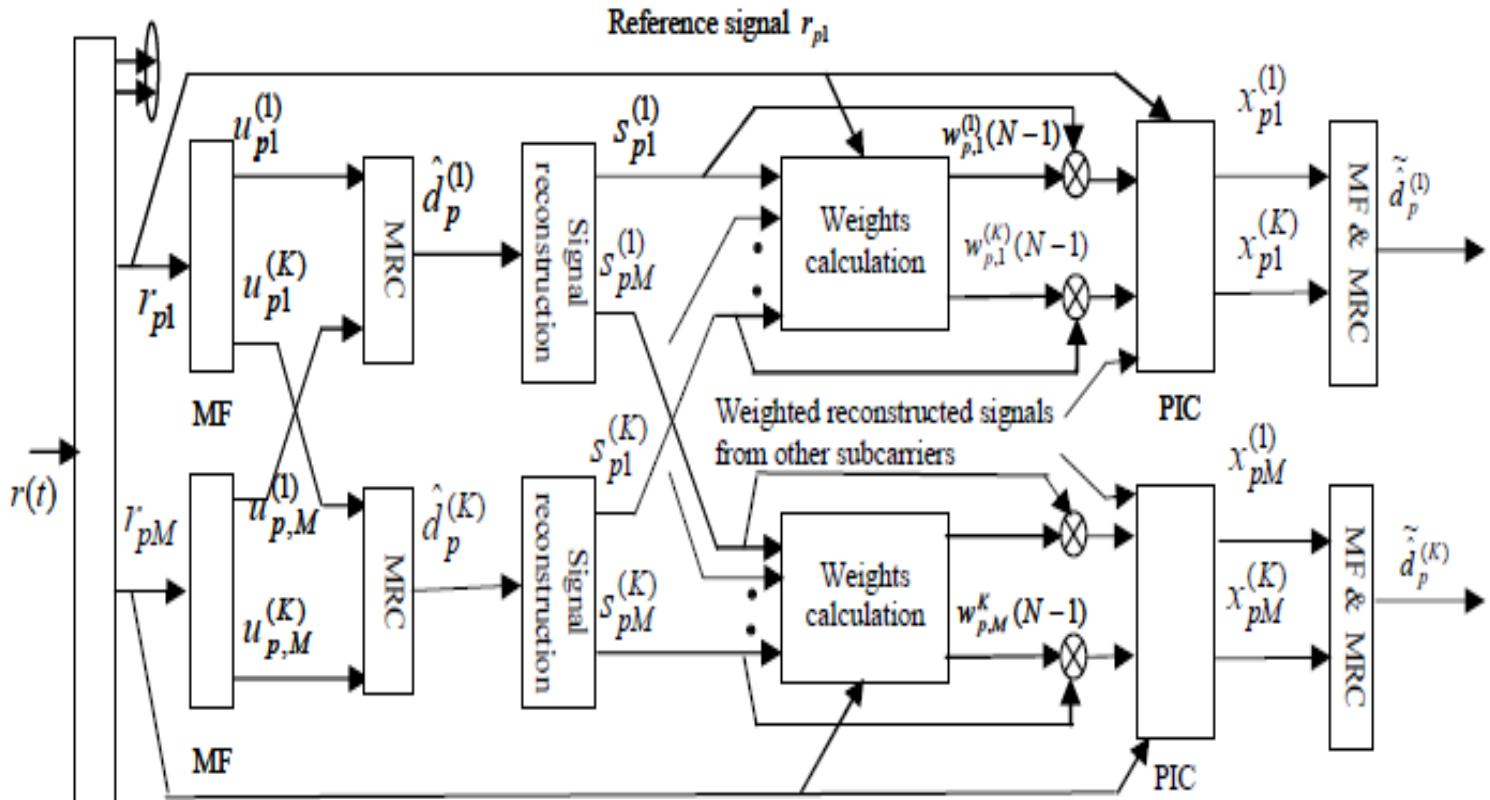

Figure.3. Block diagram for HD APIC orthogonal multicarrier DS-CDMA .[7]

Fig. 2 Block diagram for HD-APIC orthogonal multicarrier DS-CDMA receiver.[7] 


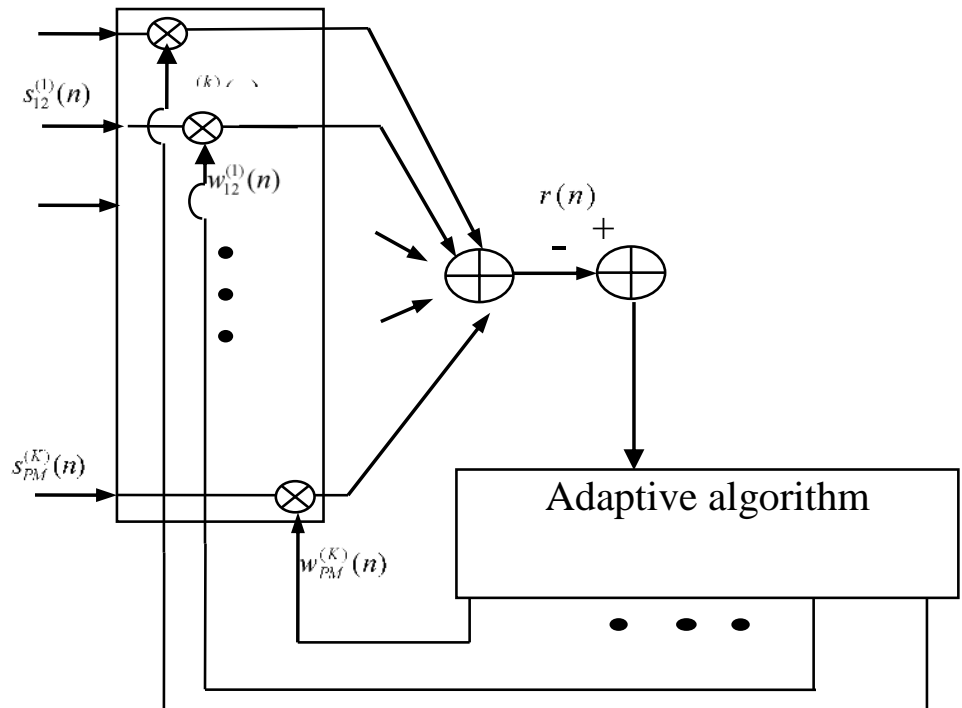

Fig. 3 Block diagram for adaptive filter as a linear combiner.

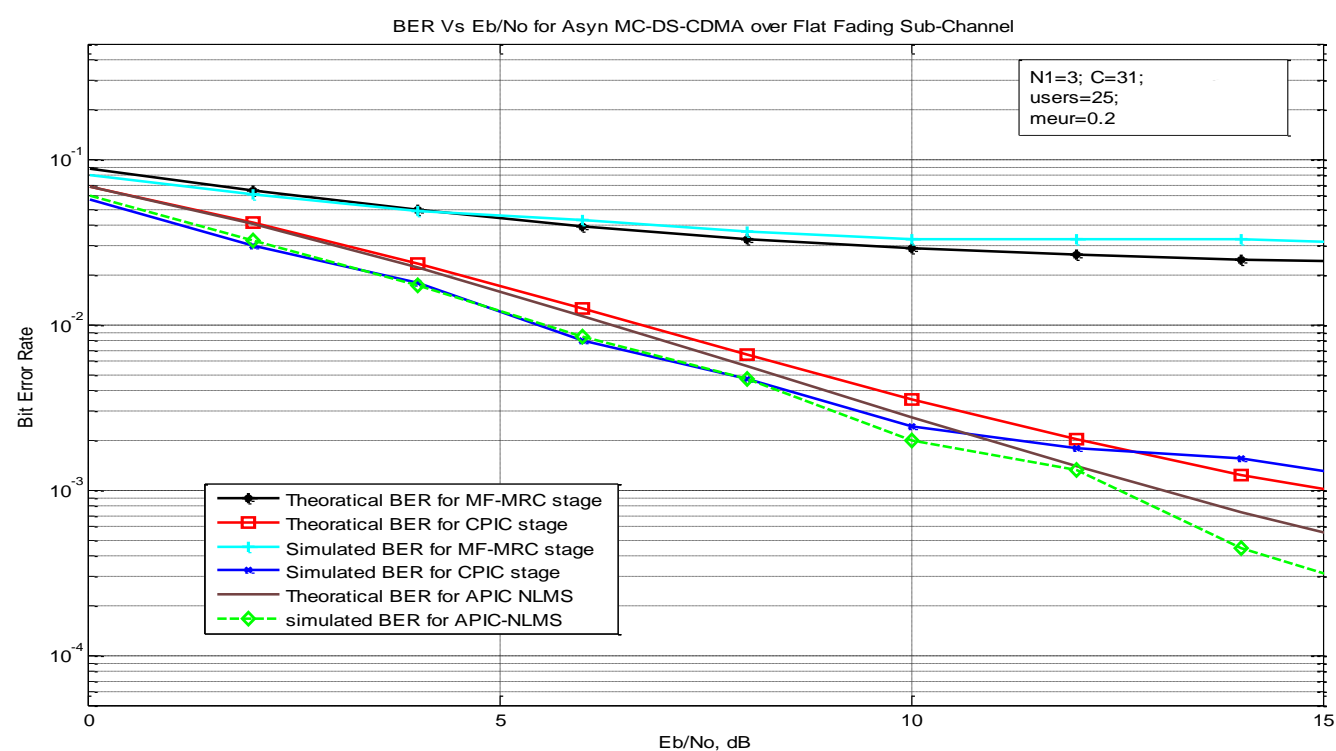

Fig. 4 BER versus of $E_{b} / N_{0}, K=15$ users, and number of sub-carriers $U=6$. 


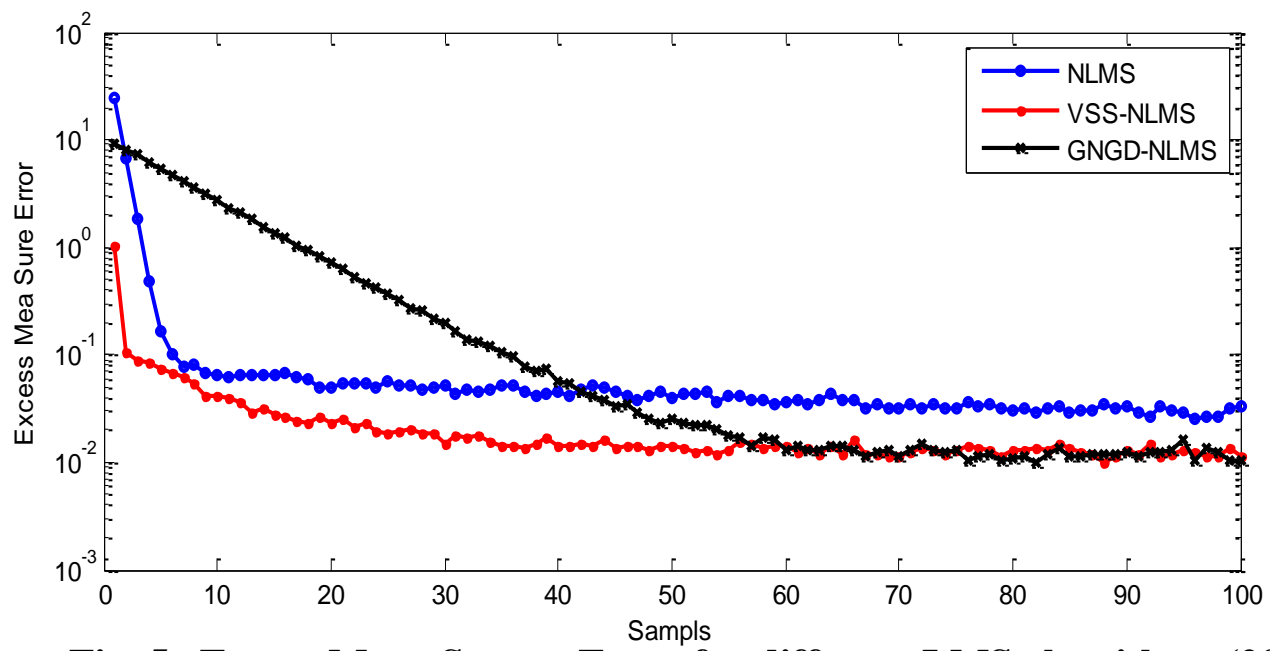

Fig. 5 Excess Mean Square Error for different LMS algorithms (20dB)

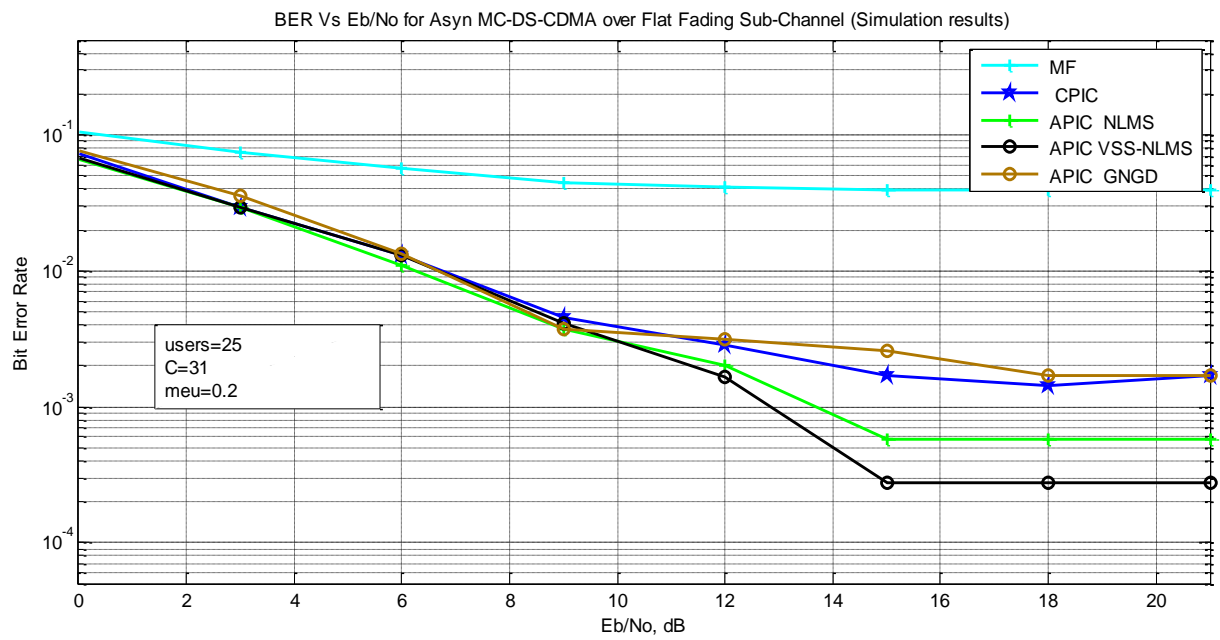

Fig. 6 BER versus of $E_{b} / N_{0}, N=31, K=25$ users, and number of sub-carriers $U=6$.

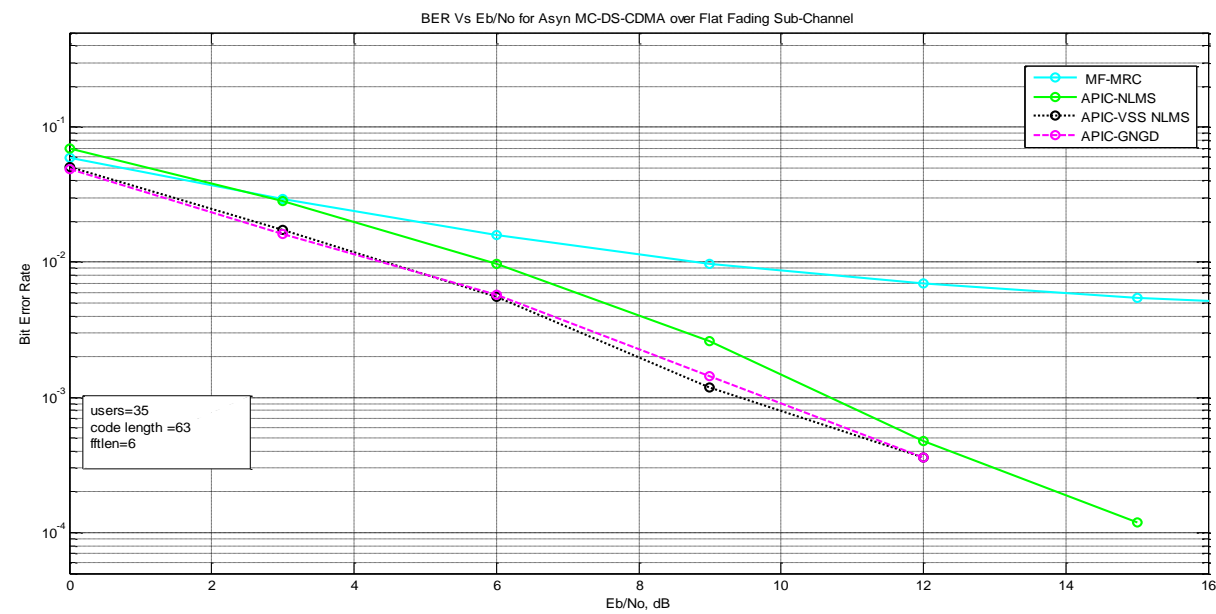

Fig. 7 BER versus of $E_{b} / N_{0}, N=63, K=35$ users, and number of sub-carriers $U=6$. 


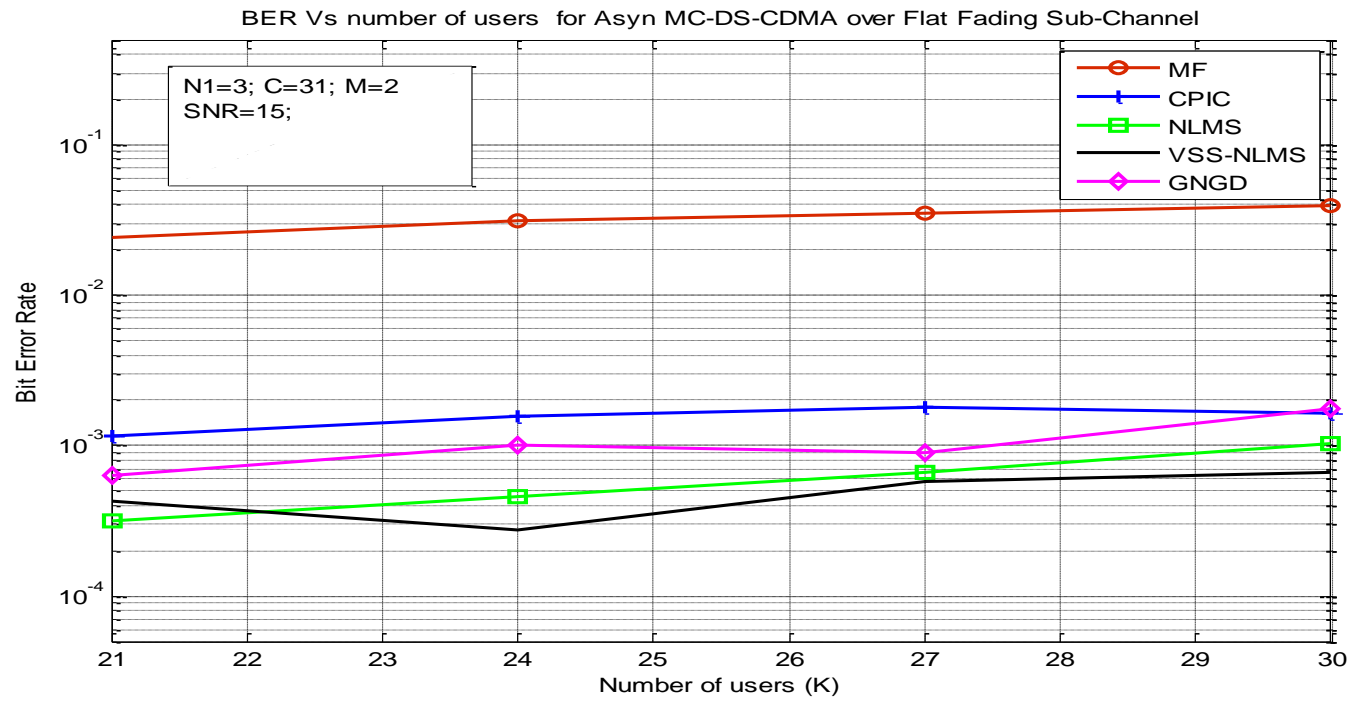

Fig. 8 BER versus of number of active users for $E_{b} / N_{0}=15 \mathrm{~dB}$, number of sub-carriers $U=6$.

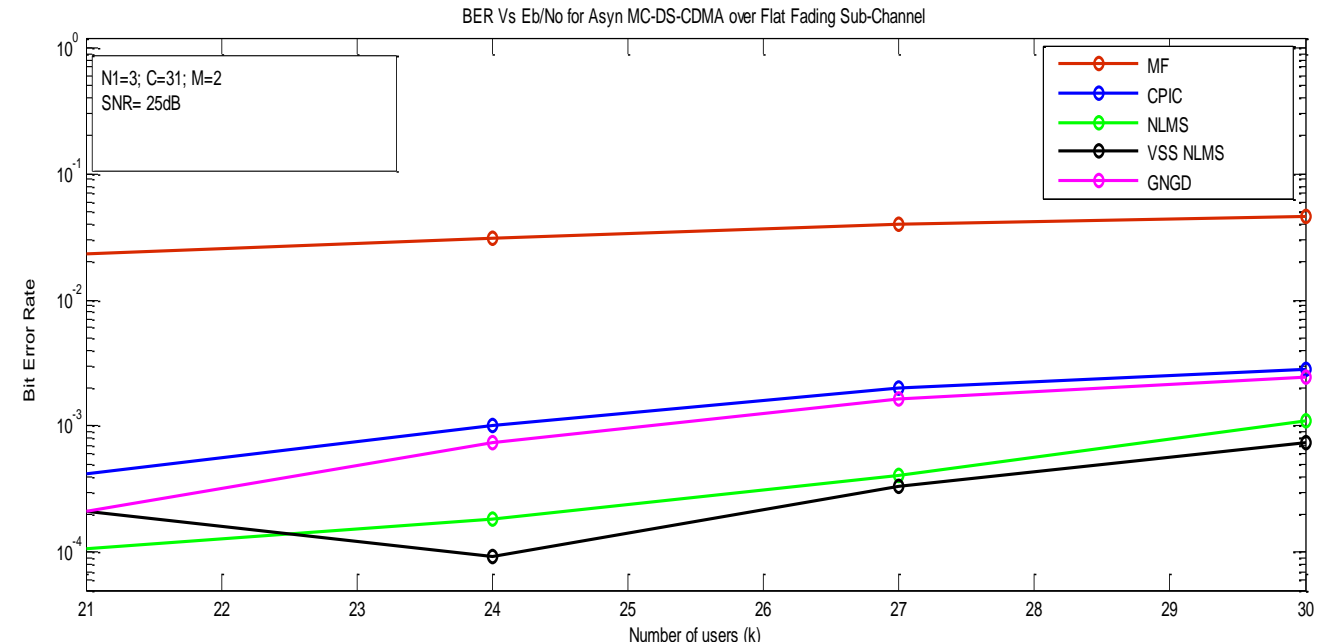

Fig. 9 BER versus of number of active users for $E_{b} / N_{o}=25 \mathrm{~dB}$, number of sub-carriers $U=6$. 